ARTICLE

\title{
Calculation of Normalised Organ and Effective Doses to Adult Reference Computational Phantoms from Contemporary Computed Tomography Scanners
}

\author{
Jan T. M. JANSEN ${ }^{*}$ and Paul C. SHRIMPTON
}

Health Protection Agency, Centre for Radiation, Chemical and Environmental Hazards, Chilton, OX11 ORQ, United Kingdom

\begin{abstract}
The general-purpose Monte Carlo radiation transport code MCNPX has been used to simulate photon transport and energy deposition in anthropomorphic phantoms due to the x-ray exposure from the Philips iCT 256 and Siemens Definition CT scanners, together with the previously studied General Electric 9800. The MCNPX code was compiled with the Intel FORTRAN compiler and run on a Linux PC cluster. A patch has been successfully applied to reduce computing times by about 4\%. The International Commission on Radiological Protection (ICRP) has recently published the Adult Male (AM) and Adult Female (AF) reference computational voxel phantoms as successors to the Medical Internal Radiation Dose (MIRD) stylised hermaphrodite mathematical phantoms that form the basis for the widely-used ImPACT CT Patient dosimetry tool. Comparisons of normalised organ and effective doses calculated for a range of scanner operating conditions have demonstrated significant differences in results (in excess of $30 \%$ ) between the voxel and mathematical phantoms as a result of variations in anatomy. These analyses illustrate the significant influence of choice of phantom on normalised organ doses and the need for standardisation to facilitate comparisons of dose. Further such dose simulations are needed in order to update the ImPACT CT Patient Dosimetry spreadsheet for contemporary CT practice.
\end{abstract}

KEYWORDS: Monte Carlo, dosimetry, computed tomography

\section{Introduction}

The International Commission on Radiological Protection (ICRP) has recommended in Publication $110^{1)}$ the adult reference computational phantoms: Adult Male (AM) and Adult Female (AF). These are voxel phantoms, based on two individuals but reconstructed to match the reference anatomical and physiological data in ICRP Publication $89,{ }^{2)}$ and are in contrast to the mathematical phantoms used previously. $^{3-6)}$ The common adoption of such specific computational phantoms should facilitate the comparison of dose between medical x-ray examinations and ensure that observed differences are due to variation in the protocols used instead of choice of dosimetric phantom.

Medical contributions to the annual effective dose per head of population have grown in recent years ${ }^{7)}$ due in part to the increase in Computed Tomography (CT) examinations and their relatively high effective doses. This pattern has resulted in some concern and also initiatives to raise awareness of the levels of patient dose. The CT Patient Dosimetry Calculator $^{8)}$ developed by the ImPACT Group (Imaging Performance Assessment of CT scanners, St Georges Hospital, London, UK) is a widely-used tool to derive typical organ and effective doses for CT protocols. It is based on organ dose coefficients (normalised to the axial CT Dose Index free-in-air (CTDI FIA $)$ ) published in 1993 as Report NRPB-SR250 ${ }^{9)}$ by the National Radiological Protection Board (NRPB) (now incorporated into the Health Protection Agency (HPA)). These data relate to the range of CT scan-

*Corresponding author, E-mail: jan.jansen@hpa.org.uk ners used in the UK in the early 1990's and were calculated utilising Monte Carlo techniques and a mathematical hermaphrodite phantom, ${ }^{4)}$ referred to as NRPB18+, which did not include all the organs at risk identified in the 2007 ICRP Recommendations ${ }^{10)}$ for the estimation of effective dose, $\mathrm{E}_{103}$.

In view of continuing developments in $\mathrm{CT}$ and the limitations of Report NRPB-SR250 in relation to updated dosimetry for contemporary CT practice, further Monte Carlo simulations are underway at HPA for a range of reference patients and scanners. In the present work, normalised organ doses have been derived for an updated mathematical hermaphrodite phantom HPA18+, together with the AM and AF voxel phantoms, for an old scanner included in Report NRPB-SR250 and two current models. Comparisons of normalised organ and effective doses are presented under a range of conditions. In addition, calculation times are reported for the various adult phantoms and the hardware and software used, following efforts to improve the speed.

\section{Materials and Methods}

\section{Hardware and Software}

A dedicated PC cluster has been used for the calculations, consisting of 11 Supermicro PC-nodes (Super Micro Computer Inc., San Jose, CA) each equipped with two Dual-Core AMD Opteron processors (Advanced Micro Devices, Dresden, Germany) of $1.8 \mathrm{GHz}$ (7 nodes) or $3.0 \mathrm{GHz}$ (4 nodes), as described in detail elseware. ${ }^{6}$ The Fedora Core Linux operating system (Fedora Core, Raleigh, NC) is installed on all the nodes with the batch system OpenPBS (Altair Engi-

(C) 2011 Atomic Energy Society of Japan, All Rights Reserved. 
neering Inc. Troy, MI) used to distribute the jobs accordingly. Radiation transport calculations are performed with the general Monte Carlo N-Particle transport code system MCNPX 2.6.0 ${ }^{11)}$ (Los Alamos National Laboratory, Los Alamos, NM). This program is compiled with the Intel FORTRAN compiler (Intel Corporation, Santa Clara, CA).

\section{Anthropomorphic Phantoms}

The reference phantoms AM and AF published by ICRP ${ }^{1)}$ have been implemented in an MCNPX input file. These phantoms have rectangular voxels with equal dimensions in the transverse plane and a larger dimension along the height of the phantom. The voxels are stacked and aligned in a regular matrix-like way. The dimensions in the transverse plane and along the height are $2.137 \mathrm{~mm}$ and $8 \mathrm{~mm}$, and $1.775 \mathrm{~mm}$ and $4.84 \mathrm{~mm}$, for the male and female phantoms, respectively. The number of voxels from right to left, from front to back and from feet to head are 254, 127 and 222, and 299, 137 and 348, for AM and AF, respectively. The option was implemented to exclude the bottom and top rows of skin in the calculations. These phantoms model the reference male and female with heights and masses of $1.76 \mathrm{~m}$ and $73 \mathrm{~kg}$, and $1.63 \mathrm{~m}$ and $60 \mathrm{~kg}$, respectively. The origins of the phantoms are located, as for the HPA18+ phantom, at the central position at the base of the trunk. This is taken to lie between the voxel slices with skin around the legs and skin around the trunk.

The ICRP publication ${ }^{1)}$ does not specify the fluence to dose functions required to calculate Red Bone Marrow (RBM) and endosteal tissue doses. These functions are necessary because secondary electron equilibrium cannot be assumed in the diagnostic x-ray energy range for these bone tissues with dimensions in the tens of micrometre range. The phantom voxel sizes are in the millimetre range, so that RBM and endosteal tissues are only available as mixtures in the spongiosa bone and, for the endosteal tissue, also in the medullary cavities of the long bones. RBM dose is calculated for each bone from the elemental composition ${ }^{12)}$ using the mass energy absorption coefficients by Seltzer ${ }^{13)}$ and applying bone-specific dose enhancement factors from King and Spiers. ${ }^{14)}$ These bone-specific RBM doses are summed, according to the RBM mass in each bone as specified for the phantoms, ${ }^{1)}$ to provide total RBM dose. The endosteal cell dose, formerly called the bone surface dose, has changed significantly in nature. It is now assumed that these cells are not contained in the cortical bone. In addition, they are localised out to 50 micrometre $^{1)}$ from both the trabecular surfaces and the interior cortical surfaces of the medullary cavities, instead of the previously assumed 10 micrometre. This invalidates the previously derived endosteal dose enhancement factors. Therefore, it is presently assumed that the endosteal cell dose is the average dose to the bone mixture of the spongiosa and medullary cavities of each bone, weighted by the endosteum mass in each bone as specified for the phantom. ${ }^{1)}$ This will probably provide an overestimate. Sex-specific normalised organ doses calculated for the $\mathrm{AM}$ and $\mathrm{AF}$ phantoms have been combined to provide sex-averaged organ doses, as specified by the ICRP. ${ }^{1)}$ These organ doses will be compared with the normalised organ doses derived for the HPA18+ phantom.

Also, the AM and AF sex specific organ doses are used separately with the tissue weighting factors in ICRP Publication $103,{ }^{10)}$ as a rough overall indicator. This extends the definition of effective dose used in this article and it differs from the sex-averaged recommendation of the ICRP. ${ }^{1,10)}$ The ICRP developed the concept of effective dose for radiation protection purposes, where the gender is generally unknown. This is a medical radiation application, where the gender is known.

The mathematical hermaphrodite phantom HPA18+ is a revised version of the MIRD-like NRPB18+ phantom $^{5)}$ that was based on the Cristy and Eckerman phantom. ${ }^{3)}$ This revision was necessary to include the oesophagus, new risk and remainder organs and to include densities and elemental compositions for additional tissues. RBM dose is calculated using the same methodology as above, except it is applied to the whole bone without distinction between cortical, spongiosa and medullary cavity segments, and the RBM masses are divided between the bones according to Cristy. ${ }^{15)}$ Endosteal cell dose is calculated as the average skeleton dose, including all mass weighted bones without further segmentation. One can expect that the RBM and endosteal cell doses are somewhat higher for the HPA18+ phantom compared with $\mathrm{AM}$ and AF due to the lack of attenuation by the outer layer of cortical bone with relatively high $\mathrm{Z}$ material and density, and the higher $\mathrm{Z}$ material content for the endosteal cells. The HPA18+ phantom models a hermaphrodite with a height of $1.74 \mathrm{~m}$ and a mass of $72.7 \mathrm{~kg}$.

The organs used in this study are the risk and remainder organs defined in ICRP Publication 103. ${ }^{10)}$ Not all these organs are available in Report NRPB SR250 and therefore the ImPACT CT Patient Dosimetry spreadsheet ${ }^{8)}$ needs to utilise surrogate organs. In addition, there are similar more minor deficiencies for the HPA18+ phantom. Surrogate organs necessarily utilised for both theses dosimetry models are listed in Table 1. For the ImPACT spreadsheet, the Rest of Body (RoB) is calculated as the mass weighted whole body dose minus all the separately named organ doses, with the exception of the eye lenses. The whole body dose is calculated as the mass weighted doses to the head, trunk and legs regions. Some of the named organs have separate wall and content masses in the spreadsheet, but the same dose is assumed. In the ImPACT spreadsheet, colon dose is calculated as a mass weighted average of doses to the Upper Large Intestine (ULI) and Lower Large Intestine (LLI). For the HPA18+ phantom, the RoB is the region inside the phantom that does not belong to a named organ or tissue.

To allow comparison between the various phantoms, normalised organ doses (relative to $\mathrm{CTDI}_{\mathrm{FIA}}$ expressed as absorbed dose to air) have been calculated for whole body examinations extending from $9 \mathrm{~cm}$ below the trunk to the top of the head. Differences in organ position are investigated through a localisation parameter, ${ }^{6)} \Delta_{o, p}$, following linear scaling of the AF phantom so that the distance from the bottom of the trunk to the top of the head matches the lengths for the NRPB18+, HPA18+ and AM phantoms. The local- 
Table 1 Surrogate organs used for dose assessment in the ImPACT spreadsheet, which uses the NRPB SR250 data employing the NRPB18+ phantom, and the HPA18+ phantom

\begin{tabular}{|c|c|c|}
\hline $\begin{array}{c}\text { Dosimetry } \\
\text { model }\end{array}$ & $\begin{array}{l}\text { Risk or remainder } \\
\text { organ }\end{array}$ & Surrogate organ \\
\hline NRPB18+ & Colon & $\begin{array}{c}\text { Mass weighted (ULI } \\
\text { + LLI) }\end{array}$ \\
\hline NRPB18+ & ET region & Thyroid \\
\hline NRPB18+ & Lymph nodes & Rest of body \\
\hline NRPB18+ & Muscle & Rest of body \\
\hline NRPB18+ & Oesophagus & Thymus \\
\hline NRPB18+ & Oral mucosa & Brain \\
\hline NRPB18+ & Prostate & Urinary Bladder \\
\hline NRPB18+ & Salivary glands & Brain \\
\hline HPA18+ & Lymph nodes & Rest of body \\
\hline HPA18+ & Muscle & Rest of body \\
\hline
\end{tabular}

isation parameter, $\boldsymbol{\Delta}_{\mathrm{o}, \mathrm{p}}$, for organ, o, and phantom, $\mathrm{p}$, is defined as:

$$
\Delta_{o, p}=\frac{1}{2}+\frac{\sum_{i=-9}^{94}\left|D_{o, p, i}-D_{o, R, i}\right|-\max \left(\sum_{i} D_{o, p, i}, \sum_{i} D_{o, R, i}\right)}{2 \min \left(\sum_{i=-9}^{94} D_{o, p, i}, \sum_{i=-9}^{94} D_{o, R, i}\right)}
$$

Where $\mathrm{D}_{\mathrm{o}, \mathrm{p}, \mathrm{i}}$ is the absorbed dose in organ, $\mathrm{o}$, in phantom, $p$, due to the $i^{\text {th }}$ slice exposure, with a slice thickness of $1 \mathrm{~cm}$ and $\mathrm{i}$ being the central beam distance in $\mathrm{cm}$ from the bottom of the trunk. The reference NRPB18+ phantom, R, organ, o, absorbed dose, $D_{o, R, i}$ due to the $i^{\text {th }}$ slab (slice) exposure with a slab width of $1 \mathrm{~cm}$ is from NRPB Report SR250, ${ }^{9)}$ as used by ImPACT CT Dosimetry ${ }^{8)}$ spreadsheet. The location parameter, $\Delta_{\mathrm{o}, \mathrm{p}}$, is always between 0 and 1 . The parameter is 0 when $\left(\mathrm{D}_{\mathrm{o}, \mathrm{p}, \mathrm{i}}-\mathrm{D}_{\mathrm{o}, \mathrm{R}, \mathrm{i}}\right)$ is non-positive or non-negative for all slabs, i, meaning a perfect match along the height of the phantom. The parameter is 1 when for all slabs, $i$, either $D_{o, p, i}$ or $\mathrm{D}_{\mathrm{o}, \mathrm{R}, \mathrm{i}}$ equal zero. This is a perfect mismatch along the height of the phantom.

\section{CT Scanners}

The General Electric 9800 CT scanner (General Electric Healthcare, Milwaukee, WI) was previously included in Report NRPB-SR250. Further simulations have been performed for an operating tube voltage of $120 \mathrm{kV}$ and $10 \mathrm{~mm}$ slice thickness for the HPA18+, AM and AF phantoms. Results are compared with those from the ImPACT CT Patient Dosimetry spreadsheet ${ }^{8)}$ for the NRPB18+ phantom, after correction of the NRPB SR250 data by a factor of $0.935^{16)}$ as the ratio of mass energy absorption coefficients for air relative to muscle.

Two examples of a contemporary CT scanner have been simulated, on the basis of technical information provided by the manufacturers: the Siemens Definition Dual Source (Siemens Medical Solutions, Forchheim, Germany) operating at four tube voltages $(80,100,120$ and $140 \mathrm{kV})$, two bow-tie filters (Body and Head), two fan beam collimations (Full and Small), and two slice thicknesses (19 and $29 \mathrm{~mm}$.); and the Philips iCT 256 (Philips Healthcare, Best, The Netherlands) operating at four tube voltages (80, 100, 120 and $140 \mathrm{kV}$ ), three bow-tie filters (Body, Head and Baby),
Table 2 Scan ranges $(\mathrm{cm})$ from base of trunk for the various phantoms for assumed typical CT examinations

\begin{tabular}{cccc}
\hline Examination & $\begin{array}{c}\text { HPA18+ } \\
(\mathrm{cm})\end{array}$ & $\begin{array}{c}\text { AM } \\
(\mathrm{cm})\end{array}$ & $\begin{array}{c}\text { AF } \\
(\mathrm{cm})\end{array}$ \\
\hline Brain & $80.54-94$ & $80-95.2$ & $70.7-86.6$ \\
Chest & $43.5-67.5$ & $44-65.6$ & $38.7-60.5$ \\
Abdomen & $22-43$ & $25.6-49.6$ & $21.3-43.6$ \\
Pelvis & $0-22$ & $4-25.6$ & $1.9-21.3$ \\
\hline
\end{tabular}

and one slice thickness of $10 \mathrm{~mm}$. The operating parameters are user selectable for a protocol. Usually the Body, Head and Baby bow-tie filters are employed in clinical protocols for adults in the body, head and cardiac region. Neither the Siemens nor the Philips scanner is presently included in the ImPACT CT Patient Dosimetry spreadsheet. ${ }^{8)}$

\section{Irradiation Geometries}

The method used to simulate each CT scanner is described in detail elsewhere. ${ }^{6}$ In short, the x-ray beam is modelled for a fixed position using the MCNPX radiation transport code and the photons hitting a cylindrical shaped gantry are written to a phase space file. A custom-made program reads the photons in the phase space file and rotates them randomly around the central axis of the cylindrical shaped gantry and writes them back into another phase space file. The second MCNPX simulation reads the photons from the rotated phase space file and transports them through the anthropomorphic and free-in-air geometries. For the anthropomorphic calculations, the x-ray beam position is varied along the central axis of the phantom in steps of $1 \mathrm{~cm}$.

\section{Examinations}

Due to the significant anatomical differences between the mathematical and voxel phantoms, specific scan ranges for typical CT examinations ${ }^{17)}$ of the brain, chest, abdomen and pelvis were defined with the help of a radiographer. Boundaries for these examinations are shown in Table 2 relative to base of trunk for the HPA18+, AM and AF phantoms.

\section{Computation Times}

There are reports ${ }^{18,19)}$ that Intel compilers are designed to slow down non-Intel processors. This is partly accomplished by comparing the processor identity response with the string GenuineIntel, whereby an AMD Opteron processor will respond with AuthenticAMD and change the software instruction pathway accordingly. Mackey ${ }^{19)}$ has written a patch that always runs the software instruction pathway as if the processor answered with GenuineIntel for both the executable and libraries. This patch has been applied throughout our system and the computing times have been compared for the HPA18+, AM and AF phantoms in relation to simulation of the Philips iCT $256 \mathrm{CT}$ scanner $(120 \mathrm{kV}$, Body filter and a $10 \mathrm{~mm}$ slice thickness located $12 \mathrm{~cm}$ above base of trunk). Timing has been observed for all nodes, except the server node, with four identical versions of MCNPX running simulations on the four cores per node. The computer times are split into set-up time, run-time and post-processing time, as described elsewhere. ${ }^{6)}$ 


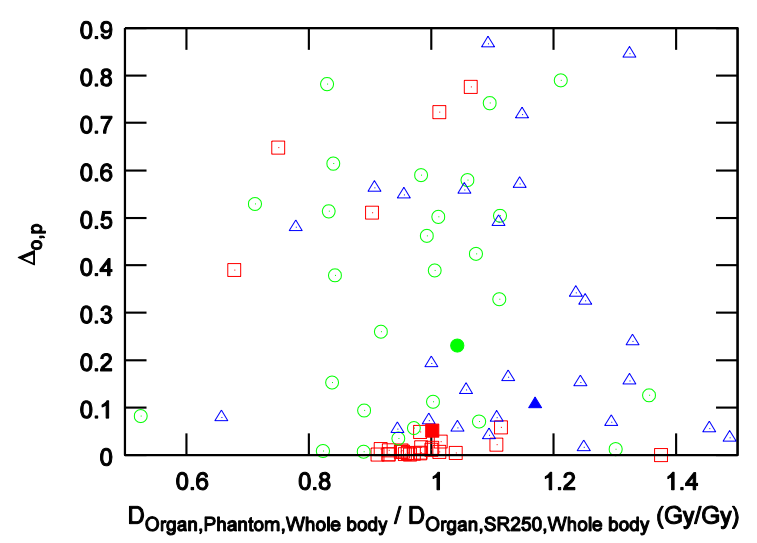

Fig. 1 Localisation parameter, $\Delta_{o, p}$, versus normalised organ dose ratio for HPA18+ $(\square)$, AM $(\circ)$ and $\mathrm{AF}_{\text {scaled }}(\Delta)$ phantoms relative to NRPB18+ for whole body examination with the GE $9800 \mathrm{CT}$ scanner $(120 \mathrm{kV})$. Effective doses $\mathrm{E}_{103}$ are marked as filled instead of open symbols.

\section{Results and Discussions}

\section{GE 9800 CT Scanner}

Normalised organ and effective doses for simulated whole body examinations of the HPA18+, AM and AF phantoms, relative to similar doses for the NRPB18+ phantom taken from Report NRPB-SR250 for the GE 9800, are shown in Fig. 1. These ratios are also plotted in relation to the localisation parameter, ${ }^{6}{ }_{\mathrm{o}, \mathrm{p}}$, for each organ, o, in order to illustrate differences in anatomy. Some significant differences in normalised organ dose are apparent between the NRPB-SR250 and new data, with deviations in excess of $30 \%$ observed in relation to the HPA18+ phantom (oesophagus (-) and thyroid (+)), AM phantom (endosteal cells, ET region (-), testes (+), and lymph nodes) and AF phantom (endosteal cells $(-)$, liver $(+)$, salivary glands, pancreas, breasts and lymph nodes) in increasing order of the ratio of whole body organ doses for the new / NRPB-SR250 data with (-) and (+) marking the border of $-30 \%$ and $+30 \%$, respectively. These differences can be at least partly attributed to use of surrogate organs (oesophagus, ET region, lymph nodes, salivary glands), tissue composition (many, especially endosteal cells), tissue density (many, especially endosteal cells) non-segmented versus segmented bones (endosteal cells), air-cavities in the head and neck region (thyroid), differences in organ depth in phantom (many, including testes and thyroid), and, for the AF phantom, differences in size (all, including liver, salivary glands, pancreas, breasts and lymph nodes).

Deviations from zero in values of the localisation parameter, ${ }^{6}{ }^{6} \Delta_{\mathrm{o} p}$, represent a mismatch in organ location. For the HPA18+ phantom, $\Delta_{o, p}$ is above 0.1 for organs where surrogates (Table 1) are exclusively assumed in NRPB18+ namely: salivary glands, oral mucosa, ET region, prostate and oesophagus, in descending order. For the voxel AM and $\mathrm{AF}_{\text {scaled }}$ phantoms, 19 and 17 organs, respectively, out of the total of 27 organs (i.e.: about $66 \%$ ) exceed this threshold, thereby demonstrating the enormous differences in anatomy

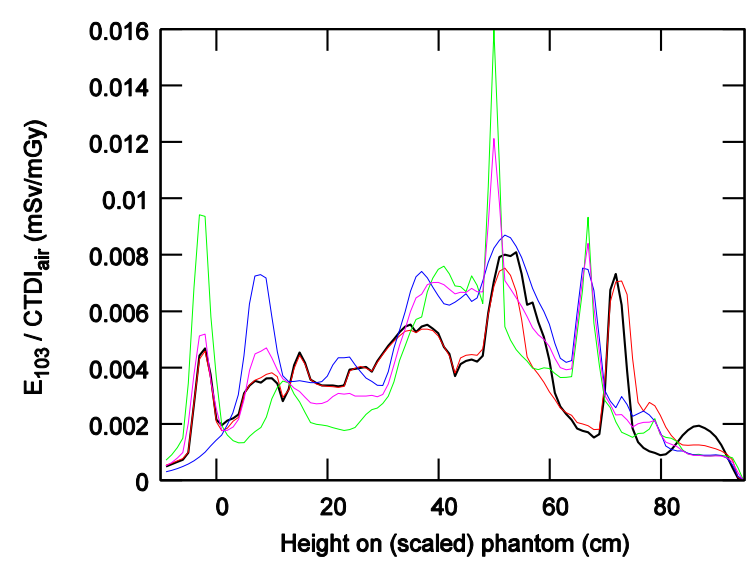

Fig. 2 The effective dose, $\mathrm{E}_{103}$, as a function of the height on the (scaled) phantom for the GE 9800 CT scanner operated at $120 \mathrm{kV}$. The colour of the line is for the NRPB18+ black, HPA18+ red, AM green, $\mathrm{AF}_{\text {scaled }}$ blue and for the sex-averaged $\mathrm{AM}+\mathrm{AF}_{\text {scaled }}$ phantom purple.

compared with the mathematical phantoms. The three largest localisation parameters, $\Delta_{\mathrm{o}, \mathrm{p}}$, in descending order are for the AM phantom: salivary glands, oral mucosa and thyroid and for the $\mathrm{AF}_{\text {scaled }}$ phantom: oral mucosa, salivary glands and thyroid. For the $\mathrm{AM}$ and $\mathrm{AF}_{\text {scaled }}$ phantoms the largest localisation parameters are observed for the NRPB-SR250 surrogate organs and the relatively small organs.

Figure 1 shows that the localisation parameter for the effective dose, $\mathrm{E}_{103}$, is larger for $\mathrm{AM}$ than for AF. To explain this fact notice that the formula for $\Delta_{\mathrm{o}, \mathrm{p}}$ can be split in three additive terms. The first term is a half, the second term is the absolute difference per slice divided by two times the minimum total dose and the third (negative) term is the maximum total dose divided by two times the minimum dose. In this case, the minimum dose is the total effective dose for NRPB18+ and the maximum dose is either the total effective dose for AM or AF. The total effective dose is just over $10 \%$ smaller for the AM compared to the AF phantom. The absolute difference per slice is shown in Fig. 2, as the area between the effective dose curves of the NRPB18+ and the other phantom. This area is more than $30 \%$ larger for the AM compared to the AF phantom. Although the difference between $\mathrm{AM}$ and $\mathrm{AF}$ is only about $0 \%$ for the constant first term, $30 \%$ for the second absolute difference term and $10 \%$ for the third term, the additive and subtractive nature of the terms in the formula for the localisation parameter, $\Delta_{\mathrm{o}, \mathrm{p}}$, makes it $110 \%$ larger for the AM compared to the AF phantom.

Figure 2 shows that the effective doses per slice for the HPA18+ and NRPB18+ phantoms are in close agreement, except in areas were the surrogate organs (Table 1) are located. This illustrate that the use of more tissue compositions and tissue densities does not have a big effect on the effective dose. Large differences in effective doses are observed for the sex specific organ regions between the hermaphrodite and sex-averaged phantoms on one side and the sex specific phantoms on the other side. The peak around $70 \mathrm{~cm}$ is for the thyroid and shows that in the mathematical phantoms it 


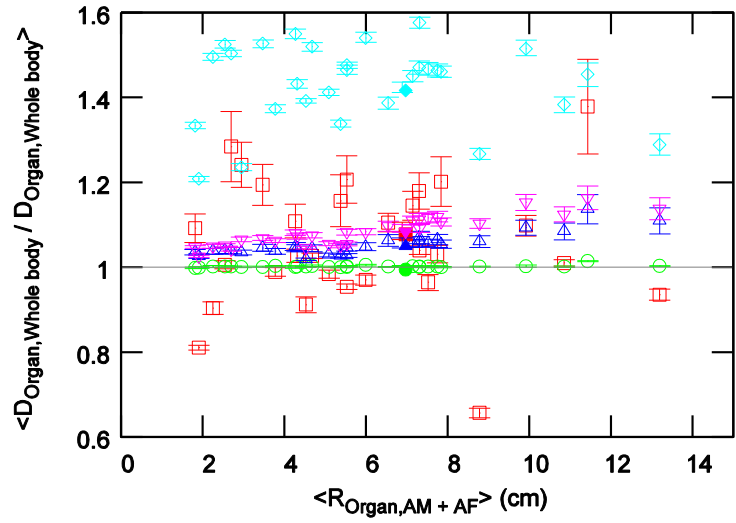

Fig. 3 Influence on normalised organ dose of changes in modelling (ICRP/ HPA18+ ( $\square$ ), $29 \mathrm{~mm} / 19 \mathrm{~mm}$ slice ( $\odot$ ), Full / Small fan $(\Delta)$, Body / Head filter $(\nabla)$ and $140 \mathrm{kV} / 80 \mathrm{kV}(\diamond))$ in relation to mean radial position of organ for whole body examination with the Siemens Definition CT scanner. Effective doses $\mathrm{E}_{103}$ at $6.9 \mathrm{~cm}$ mean radial position are marked as filled symbols and zero influence as (-).

is located at a higher position compared to the voxel phantoms. The sharp peak at $50 \mathrm{~cm}$ from the bottom of the trunk is for the AM breast organ and is not shown in the other phantoms, apart from the sex-averaged $\mathrm{AM}+\mathrm{AF}_{\text {scaled }}$ phantom, due to the modelling of the female breast only in the hermaphrodite phantoms.

Normalised effective doses, $\mathrm{E}_{103} / \mathrm{CTDI}_{100, \text { FIA }}$, for whole body examination with the GE 9800 are $0.36,0.36$ and $0.40 \mathrm{mSv} / \mathrm{mGy}$, respectively, for the NRPB18+, HPA18+ and sex-average for the AM and AF phantoms. The values for the NRPB18+ and HPA18+ phantoms are in close agreement, whereas that for the sex-average of the voxel phantoms is $10 \%$ higher.

\section{Siemens Definition CT Scanner}

Normalised organ doses have been calculated for whole body examination of the AM, AF and HPA18+ phantoms under 32 combinations of operating conditions for the Siemens Definition scanner: four tube voltages, two bow-tie filters, two fan beams and two slice thicknesses. Results for the $\mathrm{AM}$ and $\mathrm{AF}$ phantoms have been combined to derive sex-averaged organ doses. The influence on normalised organ dose of changes in phantom and operating conditions is summarised in Fig. 3, where mean values of various ratios (together with standard deviations) are plotted for each organ against its mean radial distance (radius) from the phantom central axis, $\left\langle\mathrm{R}_{\mathrm{Organ}, \mathrm{AM}+\mathrm{AF}}\right\rangle$.

For changes in phantom (HPA18+ to voxel) (red squares), dose ratios vary from 0.66 for endosteal cells to 1.37 for the breast. This behaviour illustrates the significant influence on normalised dose of choice of anthropomorphic phantom and the need for standardisation. Only larger dose ratios were observed for changes in tube voltage (from 80 to $140 \mathrm{kV}$ ).

Changes in slice thickness (from 19 to $29 \mathrm{~mm}$ ) (green circles) had little effect on dose, with ratios of normalised organ doses varying over all other conditions and phantoms between 0.9985 and 1.015, due in part to statistical uncertainties in the Monte Carlo method.

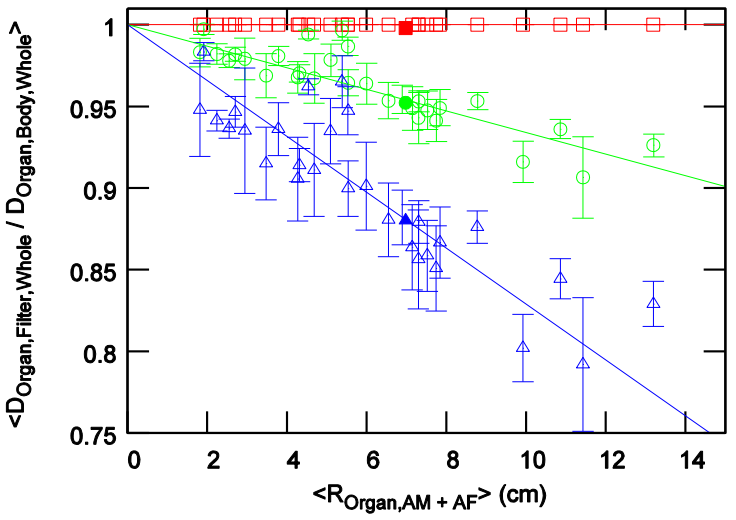

Fig. 4 Influence on normalised organ dose of changes in filter (Body $(\square)$, Head $(\circ)$ and Baby $(\Delta)$ relative to Body) in relation to mean radial position of organ for whole body examination with the Philips iCT $256 \mathrm{CT}$ scanner. Effective doses $\mathrm{E}_{103}$ at $6.9 \mathrm{~cm}$ mean radial position are marked as filled symbols.

Changes in fan beam (from small to full beam) (blue triangles) led to mean normalised organ dose ratios (over all other conditions and phantoms) between 1.019 and 1.14. The full beam gives higher normalised doses for all organs. For

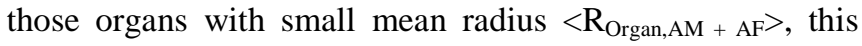
will be entirely due to scatter and for organs with large radius this will be mainly due to being out of the x-ray beam during parts of the rotation. Figure 3 shows this effect as a function of the mean organ radius $\left\langle\mathrm{R}_{\mathrm{Organ}, \mathrm{AM}+\mathrm{AF}}\right\rangle$.

For changes in filtration (from Head to Body bow-tie filter) (pink inverted triangles), mean normalised organ dose ratios lie between 1.025 and 1.17 . This effect is due to the smaller attenuation of the body bow-tie filter at larger radii and an increase in scatter. The trend with mean organ radial position is shown in Fig. 3.

Changes in tube voltage (from 80 to $140 \mathrm{kV}$ ) (blue diamonds) lead to the largest increases in normalised organ dose, with mean ratios ranging from 1.20 to 1.58 . No trend with the mean organ radius is apparent in Fig. 3. Whereas a radiation quality effect is to be expected between deep and superficial positions from an x-ray source, the rotation of the beam in CT would appear to nullify this phenomenon. Also differences in transverse dimensions between the head, neck, trunk and leg regions will disturb the relationship between superficiality and mean organ radius, thus helping to diminish the visibility of any radiation quality effect.

\section{Philips iCT 256 CT Scanner}

Normalised organ doses have been calculated for whole body examination of the AM, AF and HPA18+ phantoms under 12 combinations of operating conditions for the Philips iCT 256 CT scanner: three bow-tie filters (in increasing order of attenuation Body, Head and Baby) and four tube voltages, 80,100, 120 and $140 \mathrm{kV}$. Results for the AM and AF phantoms have been combined to derive sex-averaged normalised organ doses. The influence on normalised organ dose of changes in filter is summarised in Fig. 4, where mean values of dose relative to those for the Body filter (together with standard deviations) are plotted for each organ 
Table 3 Typical normalised effective doses, $\mathrm{E}_{103} / \mathrm{CTDI}_{\mathrm{FIA}}$, calculated for standard examinations for the HPA18+, AM and AF phantoms with the Siemens Definition and Philips iCT 256 CT scanners.

\begin{tabular}{ccccc}
\hline Examination & Scanner & \multicolumn{3}{c}{$\mathrm{E}_{103} / \mathrm{CTDI}_{\mathrm{FIA}}(\mathrm{mSv} / \mathrm{mGy})$} \\
& & HPA18+ & AM & AF \\
\hline Brain & Definition & 0.022 & 0.020 & 0.026 \\
Brain & iCT 256 & 0.026 & 0.024 & 0.031 \\
Chest & Definition & 0.16 & 0.19 & 0.23 \\
Chest & iCT 256 & 0.21 & 0.24 & 0.27 \\
Abdomen & Definition & 0.15 & 0.20 & 0.19 \\
Abdomen & iCT 256 & 0.18 & 0.25 & 0.23 \\
Pelvis & Definition & 0.11 & 0.071 & 0.13 \\
Pelvis & iCT 256 & 0.13 & 0.084 & 0.15 \\
\hline
\end{tabular}

against its mean distance (radius) from the phantom central axis, $\left\langle\mathrm{R}_{\mathrm{Organ}, \mathrm{AM}+\mathrm{AF}}\right\rangle$. Normalised doses for all organs decrease when changing from Body via Head to Baby bow-tie filtration. These trends are apparent in Fig. 4, where dose ratios (for Head and Baby compared with the Body filter) broadly decrease linearly with increasing mean organ radius. Organs that are significantly above these linear trends are identified as organs in the head, or organs distributed between the head, trunk and leg regions. Although for a fixed point in the head the direct attenuation of the beam by a particular bow-tie filter will be the same as for that point in the body, the scatter and the depth of this point will both differ and this will affect the dose.

\section{Examinations}

Normalised effective doses, $\mathrm{E}_{103} / \mathrm{CTDI}_{\mathrm{FIA}}$, for the examinations defined in Table 2 for the mathematical HPA18+ and the voxel AM and AF phantoms are summarised in Table 3 for the Philips iCT 256 and Siemens Definition CT scanners (both operated at $120 \mathrm{kV}$, appropriate bow-tie filter, Full fan beam and $10 \mathrm{~mm}$ slice thickness). Coefficients are on average higher for the AF than the HPA18+ phantom by $19 \%$, $35 \%, 30 \%$ and $21 \%$ for brain, chest, abdomen and pelvis examinations, respectively, whereas in a similar comparison those for the AM are lower for brain $(-7 \%)$ and pelvis $(-34 \%)$ but higher for chest $(16 \%)$ abdomen $(36 \%)$. Half of these differences exceed $30 \%$ and therefore the influence of phantom selection on normalised dose can be called large. Normalised effective doses are between $16 \%$ and $28 \%$ larger for the Philips iCT 256 compared with the Siemens Definition CT scanner. Although these doses are scanner dependent, absolute doses for specific examinations will also depend on the protocol used.

\section{Computation Times}

Set-up and run-times are reported in Table 4 as means and their standard errors for the Intel compiled MCNP executables both with and without the additional patch. Separate values are shown for nodes with processor clock frequencies of $1.8 \mathrm{GHz}$ and $3.0 \mathrm{GHz}$, and for the HPA18+, $\mathrm{AM}$ and $\mathrm{AF}$ phantoms, with at least 8 timings being available. Post-processing time is not taken into account since times were 0.01 minutes or less and were affected by round-
Table 4 Means and their standard errors for set-up and run-times evaluated for the Intel compiled MCNPX (ifce) and patched (patch) code for single slice exposures of the HPA18+ $\mathrm{AM}$ and AF phantoms with the Philips iCT $256 \mathrm{CT}$ scanner.

\begin{tabular}{ccccc}
\hline code & $\begin{array}{c}\text { Processor } \\
\text { frequency } \\
(\mathrm{GHz})\end{array}$ & Phantom & $\begin{array}{c}\text { Set-up } \\
\text { time (s) }\end{array}$ & Run time (s) \\
\hline ifce & 1.8 & HPA18+ & $7.1 \pm 0.3$ & $160.7 \pm 0.3$ \\
patch & 1.8 & HPA18+ & $6.5 \pm 0.1$ & $152.6 \pm 0.2$ \\
ifce & 1.8 & AM & $36.3 \pm 0.4$ & $723 \pm 4$ \\
patch & 1.8 & AM & $34.6 \pm 0.2$ & $688 \pm 5$ \\
ifce & 1.8 & AF & $51.9 \pm 0.6$ & $822 \pm 3$ \\
patch & 1.8 & AF & $50.9 \pm 0.6$ & $799 \pm 8$ \\
ifce & 3.0 & HPA18+ & $4.6 \pm 0.2$ & $95.9 \pm 0.1$ \\
patch & 3.0 & HPA18+ & $4.2 \pm 0.2$ & $91.3 \pm 0.1$ \\
ifce & 3.0 & AM & $21.9 \pm 0.2$ & $430 \pm 2$ \\
patch & 3.0 & AM & $21.0 \pm 0.2$ & $416 \pm 2$ \\
ifce & 3.0 & AF & $31.8 \pm 0.3$ & $495 \pm 1$ \\
patch & 3.0 & AF & $31.1 \pm 0.2$ & $479 \pm 2$ \\
\hline
\end{tabular}

ing in MCNPX output.

The patch reduced computer times for set-up by about 5\% (partly significant) and for run-times by $4 \%$ (highly significant). The large uncertainties in the set-up times are due in part to rounding of the reported small computer times and the fact that the Linux operating system is multi-tasking such that it is not guaranteed that the core performs only MCNPX calculations. Although in principle this should not affect the reported time, it will affect the data stored in cache and this will affect the reported times.

The expected reduction in computer time calculated for a change in processor frequency from $1.8 \mathrm{GHz}$ to $3.0 \mathrm{GHz}$ is $40 \%$. This is very close to the actual reduction observed, especially for the run time. This behaviour supports the application being calculation intensive, although other components, such as the mother board, will also have an influence on the reported times.

Set-up times for the AM and AF phantoms were 5 and 7 times longer, respectively, than for the HPA18+ phantom, with run times being 4.5 and 5.2 times longer, respectively. Whereas the AM phantom has larger dimensions compared with the AF phantom and therefore higher probability for interactions and demands for increased computer time, it also has less voxels and therefore less voxel crossings with lower demand for computer time. In practice, run-times shown in Table 4 for these phantoms scale with the mean number of voxels in one dimension in the transverse plane.

\section{Conclusion}

The ICRP-110 AM and AF phantoms have been implemented in MCNPX format and an operating patch applied to reduce computing times by about $4 \%$. Normalised organ doses calculated for these voxel phantoms and a revised mathematical phantom HPA18+ have been calculated for whole body examination with the GE $9800 \mathrm{CT}$ scanner and compared with data published previously in Report NRPB-SR250 for the NRPB18+ mathematical phantom. 
Detailed analyses demonstrate significant differences (in excess of 30\%) in normalised dose between the voxel and mathematical phantoms as a result of variations in anatomy. Further calculations for the Siemens Definition and Philips iCT scanners under a range of operating conditions illustrate the significant influence of choice of phantom on normalised organ doses and the need for standardisation to facilitate comparisons of dose. Normalised effective doses $\mathrm{E}_{103} / \mathrm{CTDI}_{\mathrm{FIA}}$ for the AF voxel phantom were 19 to $35 \%$ larger than those for HPA18+ for typical examinations of the brain, chest, abdomen and pelvis. Similar comparisons for $\mathrm{AM}$ revealed relatively lower normalised doses (by -7 to $-34 \%$ ) for examinations of the brain and pelvis, but higher (by 16 to 36\%) for the chest and abdomen. Normalised effective doses for the Philips iCT 256 were $16 \%$ to $28 \%$ larger than those for the Siemens Definition scanner, although absolute doses for specific examinations will be determined by the particular exposure settings used. Further such dose simulations are needed in order to update the ImPACT CT Patient Dosimetry spreadsheet ${ }^{8)}$ for contemporary practice.

\section{Acknowledgments}

The authors thank Sally MacLachlan (HPA) for advising on the boundaries of typical CT examinations and the manufacturers for kindly supplying details of the CT scanners under Non-Disclosure Agreements.

\section{References}

1) International Commission on Radiological Protection, "Adult reference computational phantoms," ICRP Publication 110. Ann. ICRP, 39 [2], 1-165 (2009).

2) International Commission on Radiological Protection, "Basic anatomical and physiological data for use in radiological protection: reference values," ICRP Publication 89. Ann. ICRP, 32 [3-4], 1-277 (2002).

3) M. Cristy, K. F. Eckerman, Specific absorbed fractions of energy at various ages from internal photon sources. I Methods, ORNL/TM-8381/V1, Oak Ridge National Laboratory (ORNL) (1987).

4) D. G. Jones, B. F. Wall, Organ doses from medical $x$-ray examinations calculated using Monte Carlo techniques, NRPB-R186. National Radiological Protection Board, Chilton, UK (1985).

5) A. Khursheed, M. C. Hillier, P. C. Shrimpton, B. F. Wall, "In- fluence of patient age on normalized effective doses calculated for CT examination," Br. J. Radiol., 75, 819-830 (2002).

6) J. T. M. Jansen, P. C. Shrimpton, M. Zankl, "Development of PC based Monte Carlo simulations for the calculation of scanner-specific normalized organ doses from CT," International Conference on Mathematics, Computational Methods \& Reactor Physics, Saratoga Springs, New York, May 3-7, 2009, 1-15 (2009, [CD-ROM].

7) National Council on Radiation Protection and Measurements (NCRP), Ionizing radiation exposure of the population of the United States, NCRP Report 160, Bethesda, MD. (2009).

8) Imaging Performance Assessment of CT scanners (ImPACT), "ImPACT CT patient dosimetry calculator," http://www.impactscan.org/ctdosimetry.htm

9) D. G. Jones, P. C. Shrimpton, Normalised organ doses for $x$-ray computed tomography calculated using Monte Carlo techniques, NRPB-SR250, National Radiological Protection Board, Chilton, UK (1993).

10) International Commission on Radiological Protection, "The 2007 recommendations of the ICRP," ICRP Publication 103, Ann. ICRP, 37, [2-4], 1-332 (2008).

11) D. B. Pelowitz, MCNPX user's manual, version 2.6.0, LA-CP-07-1473, Los Alamos National Laboratory (LANL) (2005).

12) International Commission on Radiation Units and Measurements, Photon, electron, proton and neutron interaction data for body tissues, ICRU Report 46, Bethesda, MD (1992).

13) S. M. Seltzer, "Calculation of photon mass energy-transfer and mass energy-absorption coefficients," Radiat. Res., 136, 147-170 (1993).

14) S. D. King, F. W. Spiers, "Photoelectron enhancement of the absorbed dose from $\mathrm{X}$ rays to human bone marrow: experimental and theoretical studies," Br. J. Radiol., 58, 345-356 (1985).

15) M. Cristy. "Active bone marrow distribution as a function of age in humans," Phys. Med. Biol., 26, 389-400 (1981).

16) P. C. Shrimpton, D. G. Jones, M. C. Hillier, B. F. Wall, J. C. Le Heron, K. Faulkner, Survey of CT practice in the UK. Part 2: Dosimetric aspects, NRPB-R249. National Radiological Protection Board, Chilton, UK (1991).

17) G. Bongartz, S. J. Golding, A. G. Jurik, et al. European guidelines on quality criteria for computed tomography, Report EUR 16262 (1999).

18) J. Leibowitz, P. J. Harbour, W. E. Kovacic, J. T. Rosch, United States of America before the Federal Trade Commission, Docket No. 9341, (2009).

19) M. D. Mackey, S. Mackey. "Intel's compiler: is crippling the competition acceptable?" http://www.swallowtail.org/ naughty-intel.shtml (2005). 